Supporting Information

\title{
Meniscus-Guided 3D Microprinting of Pure Metal-Organic Frameworks with High Gas-Uptake Performance
}

Yu Liul, ${ }^{1,+,}$, Jihyuk Yangl, ${ }^{1,}$, Chaoran Tao ${ }^{2}$, Heekwon Lee ${ }^{l}$, Mojun Chen ${ }^{1}$, Zhaoyi Xu ${ }^{1}$, Huiren

Peng ${ }^{2}$, Xiao Huan ${ }^{1}$, Jie Li ${ }^{2}$, Xing Cheng ${ }^{2, *}$, Ji Tae Kim ${ }^{1, *}$

${ }^{1}$ Department of Mechanical Engineering, The University of Hong Kong, Pokfulam Road, Hong

Kong, China

${ }^{2}$ Department of Materials Science and Engineering, Southern University of Science and

Technology, Shenzhen 518055, China

Corresponding authors:

1,*Email: jtkim@hku.hk

2,*Email: chengx@ sustech.edu.cn

Y.L. and J.Y. contributed equally to this work. 

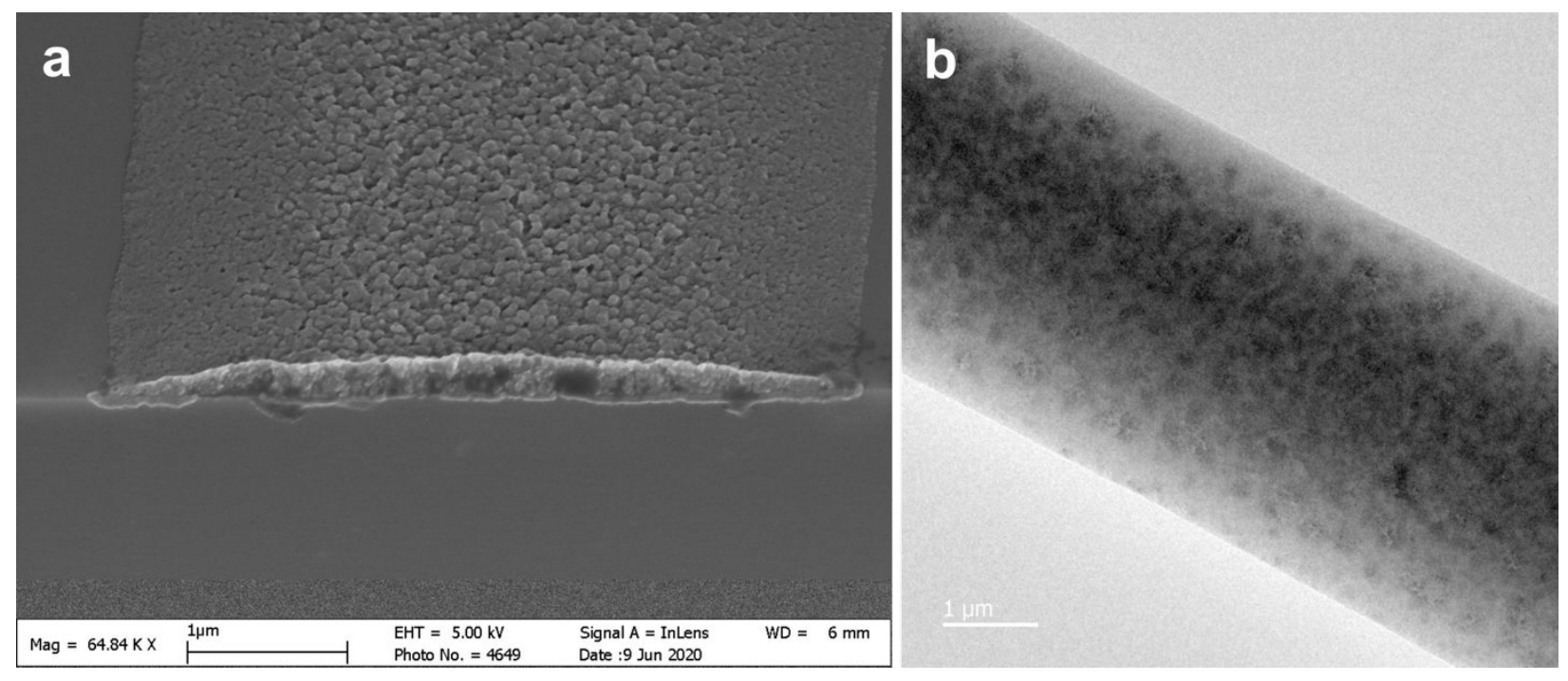

Figure S1. a) FE-SEM image of a printed HKUST-1 micro-line. b) TEM image of a printed HKUST-1 micro-line composed of nanocrystals. 


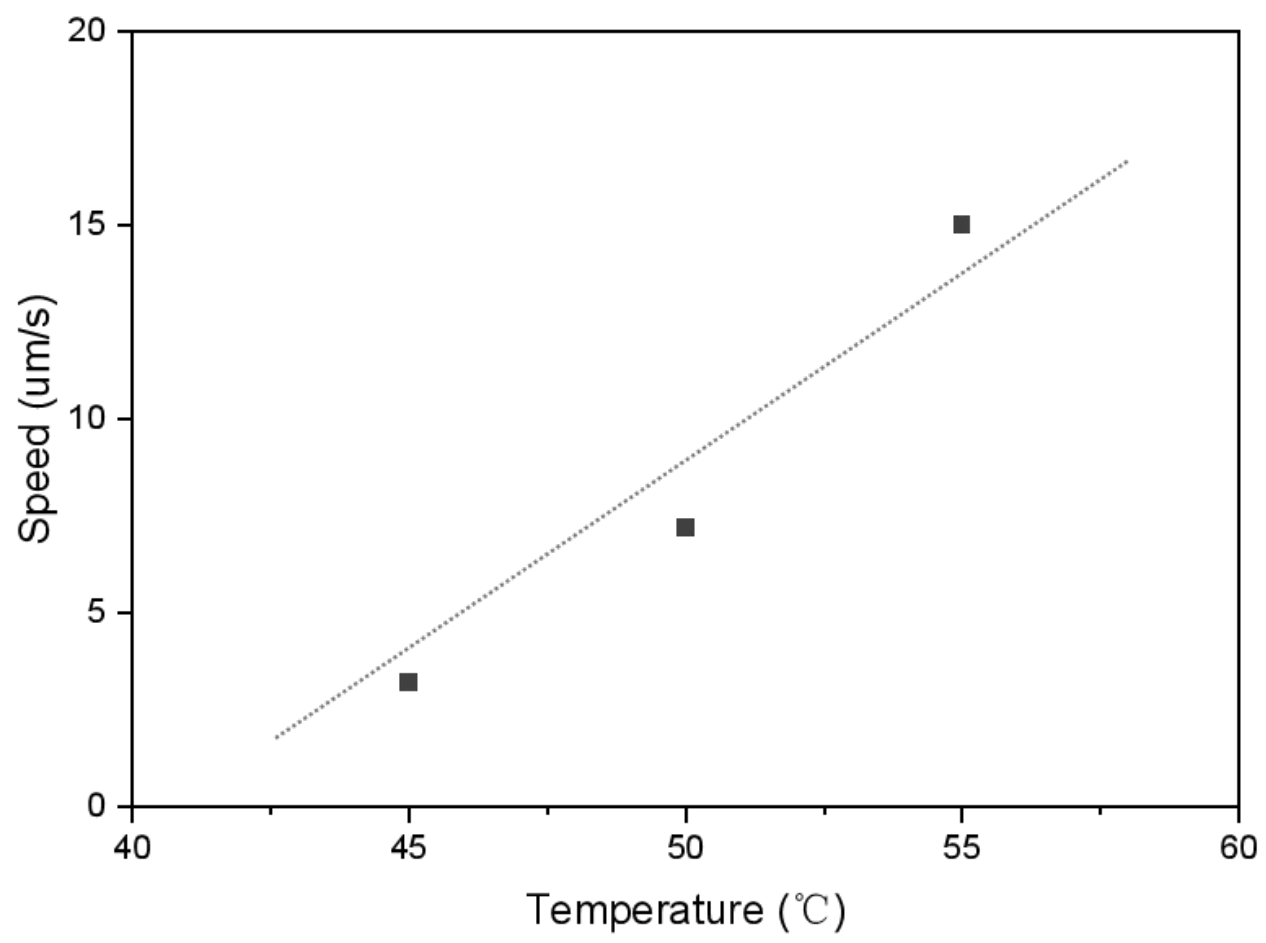

Figure S2. Printing speed of the minimum printing thickness versus temperature.

a
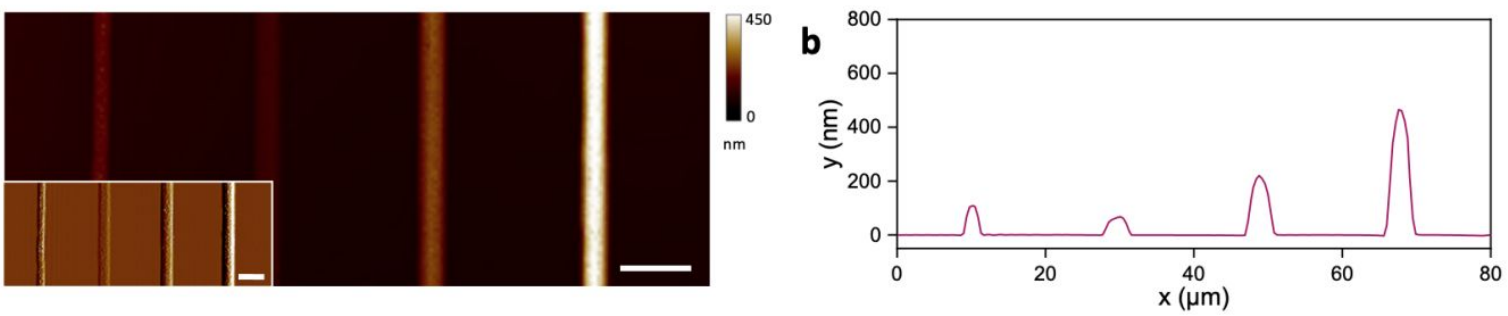
Figure S3. a) AFM topographic image and b) corresponding height profile of printed HKUST-1 micro-lines consisting of 1, 2, 5, and 10 layers. (scale bars: $10 \mu \mathrm{m}$ )

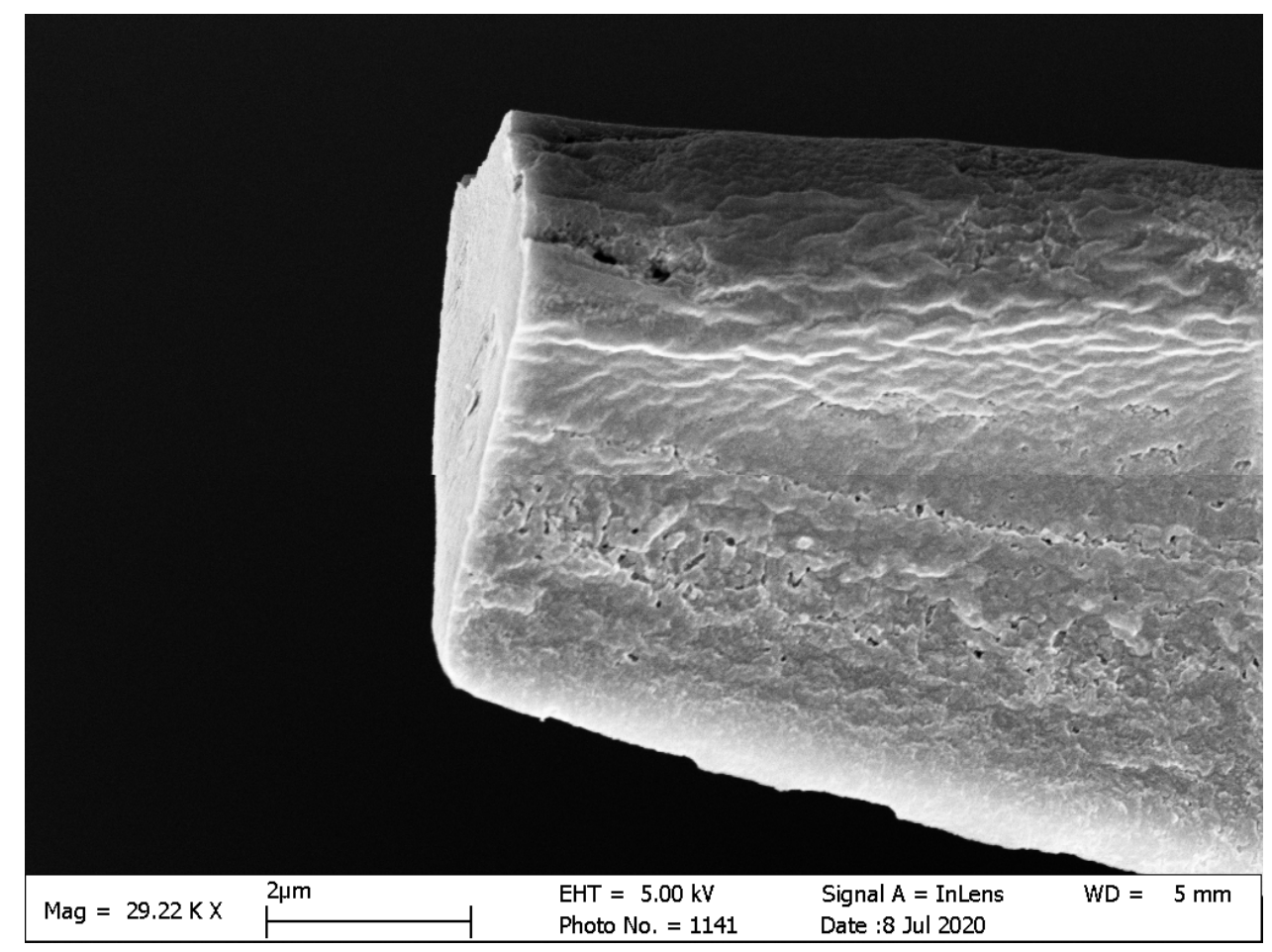

Figure S4. FE-SEM image of a 3D printed HKUST-1 micro-wall. 

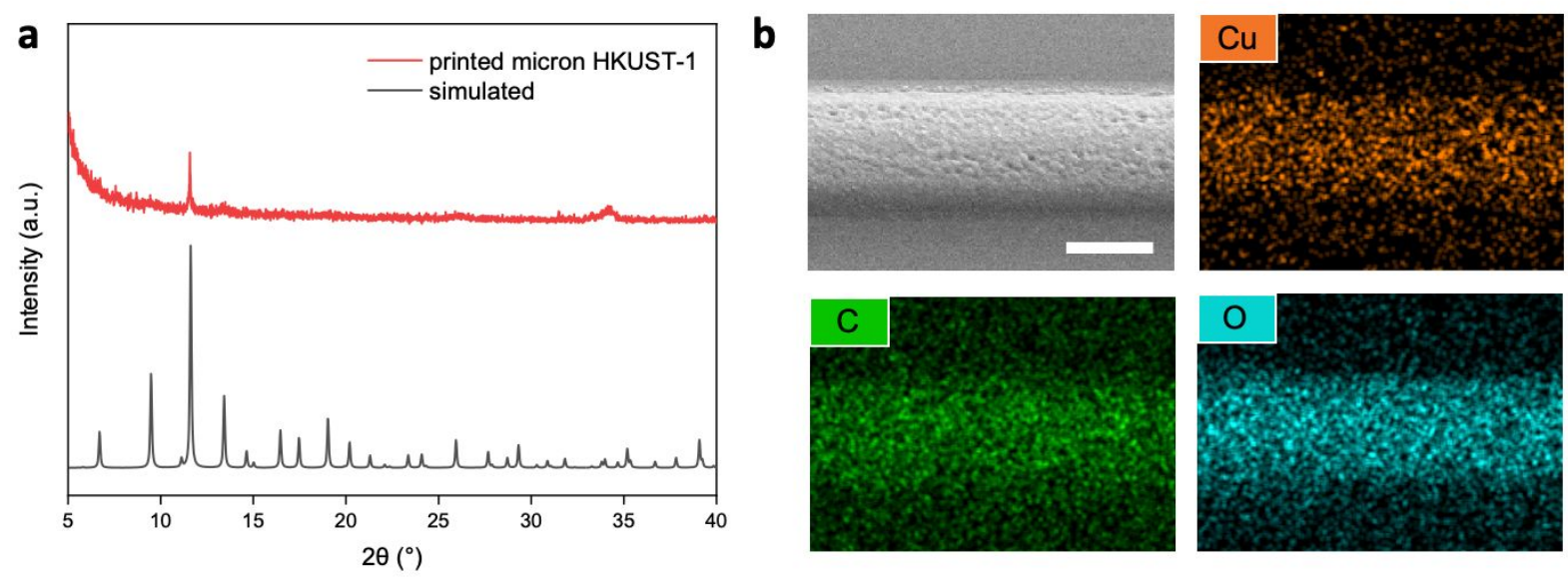

Figure S5. a) X-ray diffraction pattern of a printed HKUST-1 micro-line (red; upper) and simulated data (gray; lower). b) Energy-dispersive X-ray spectroscopy (EDX) images of the printed HKUST-1 micro-line showing the distributions of $\mathrm{Cu}$ (orange), $\mathrm{C}$ (green), and $\mathrm{O}$ (light blue). (scale bar: $2 \mu \mathrm{m}$ ). 


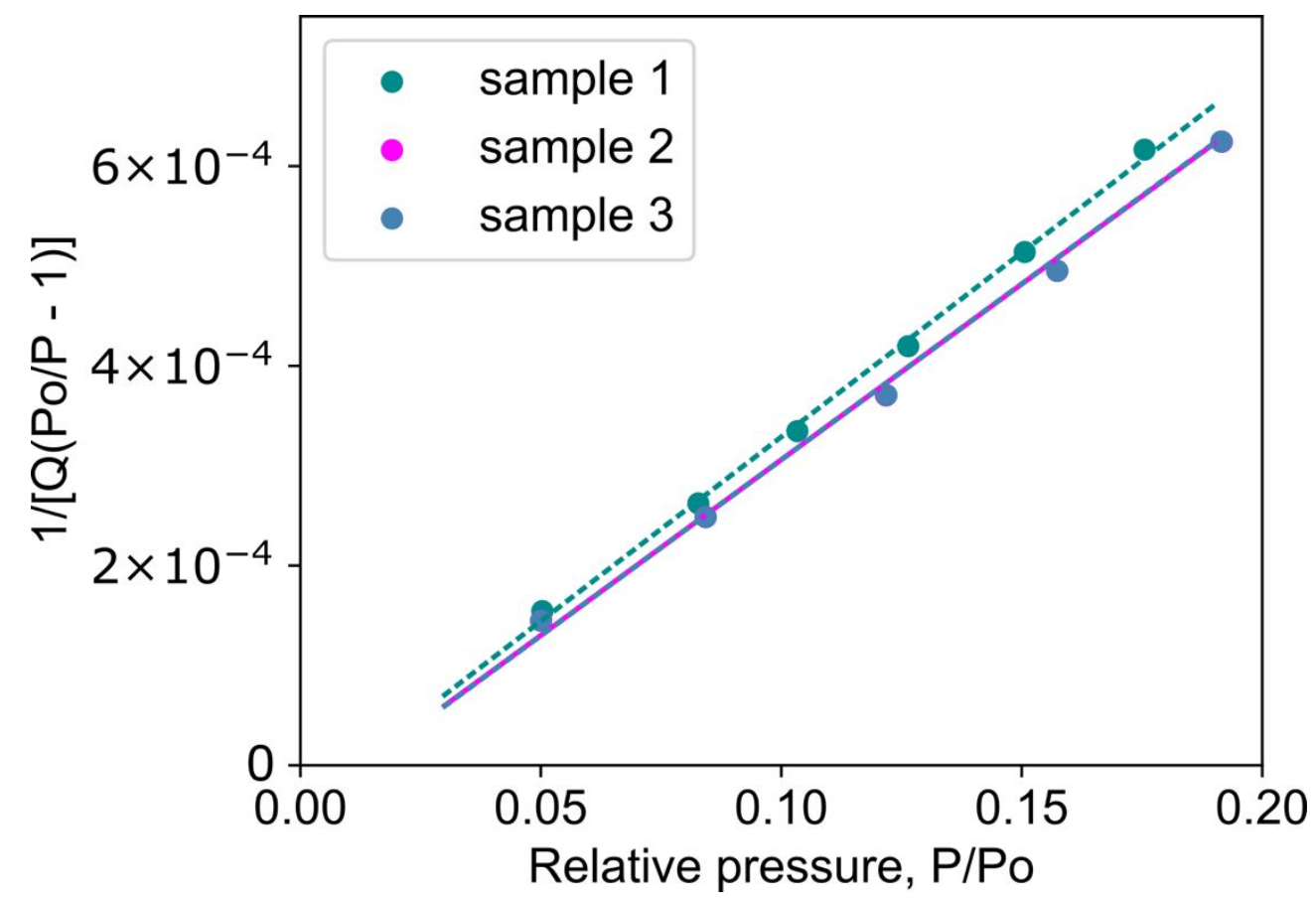

Figure S6. Brunauer-Emmett-Teller (BET) plots of the relative pressure range chosen for the determination of the BET surface area.
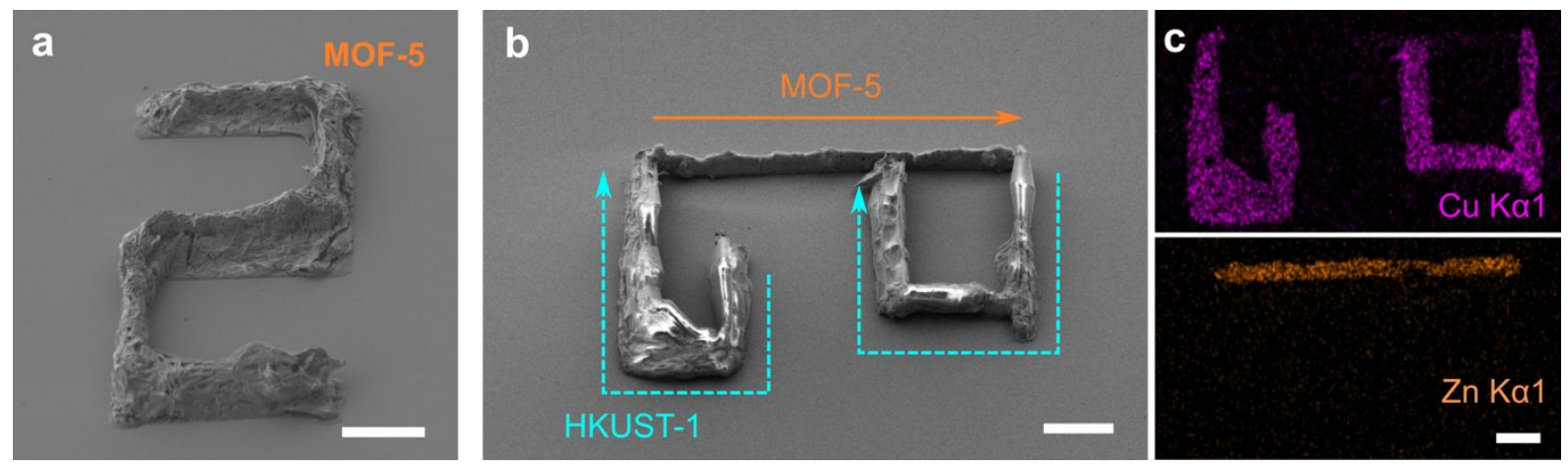

Figure S7. a) FE-SEM image of a 3D printed MOF-5 structure with a serpentine shape. b) FESEM image of a 3D printed heterostructure consisting of HKUST-1 and MOF-5, and c) the corresponding EDX image. (scale bars: $20 \mu \mathrm{m}$ ) 